Çukurova Üniversitesi Mühendislik Mimarlık Fakültesi Dergisi, 35(3), ss. 659-668, Eylül 2020

\title{
Bir Top ve Çubuk Sisteminin Ayrıklaştırılmış Geri Adımlamalı Kayan Kipli Kontrolï
}

\author{
$\operatorname{Barış~ATA*1~}$ \\ ${ }^{1}$ Çukurova Üniversitesi, Mühendislik Fakültesi, Bilgisayar Mühendisliği Bölümü, Adana
}

Geliş tarihi: 22.09 .2020

Kabul tarihi: 23.10 .2020

Öz

$\mathrm{Bu}$ çalışmada, eksik eyleyicili mekanik sistemlerin bir örneği olan top ve çubuk sistemi için bir ayrıklaştırılmış geri adımlamalı kayan kipli kontrol kuralı tasarlanmıştır. Kayan kipli kontrol ve geri adımlamalı kontrol yöntemlerini birleştiren ve bir ayrıklaştırma algoritması ile bu yöntemin eksik eyleyicili sistemlere uygulanmasını sağlayan ayrıklaştırılmış geri adımlamalı kayan kipli kontrol yöntemi top ve çubuk sisteminin kontrolünde kullanılmıştır. Kontrolörün tasarım yöntemi Lyapunov teoremine dayandığı için sistemin kararlılığı garanti altına alınmıştır. Tasarlanan kontrolörün etkinliği benzetim çalışmaları ile test edilmiştir. Benzetim bulguları ayrıklaştırılmış geri adımlamalı kayan kipli kontrol yönteminin geleneksel ayrıklaştırılmış kayan kipli kontrol yöntemi ile karşılaştırıldığında dış bozuculara karşı etkili bir kontrol sinyali ürettiğini göstermiştir.

Anahtar Kelimeler: Eksik eyleyicili mekanik sistemler, Top ve çubuk sistemi, Kayan kipli kontrol, Geri adımlamalı kontrol

\section{Decoupled Backstepping Sliding Mode Control of a Ball and Beam System}

\begin{abstract}
In this study, a decoupled backstepping sliding mode controller is designed for a ball and beam system as an example of underactuated mechanical systems. The decoupled backstepping sliding mode control method which is a combination of backstepping and sliding mode with a decoupling algorithm is considered to control a ball and beam system. The stability of the system is guaranteed by the design procedure of the controller which is based on the Lyapunov theorem. The effectiveness of the designed controller is verified by simulation tests. Simulation results show that the decoupled backstepping sliding method provides a robust control signal under external disturbances compared to the conventional decoupled sliding mode control method.
\end{abstract}

Keywords: Underactuated mechanical systems, Ball and beam system, Sliding mode control, Backstepping control

*Sorumlu yazar (Corresponding author): Barış ATA, bata@cu.edu.tr 


\section{GIiRiş}

Serbestlik derecesinden daha az sayıda eyleyiciye sahip olan sistemler eksik eyleyicili sistemler olarak tanımlanır. Eksik eyleyicili sistemler daha az sayıda eyleyici ile kontrol edildiklerinden maliyetin ve sistem karmaşıklığının azaltılması amacıyla robotik uygulamaları [1], su altı araçları [2] ve uzay araçları [3] gibi gerçek uygulamalarda da kullanılmaktadır. Bu nedenle eksik eyleyicili mekanik sistemlerin kontrolü günümüzde de aktif bir çalışma alanı olmaya devam etmektedir [4-6]. Ancak eksik eyleyicili mekanik sistemlerin kontrolü tam eyleyicili sistemlere göre daha karmaşık bir problem ortaya koymaktadır. $\mathrm{Bu}$ nedenle arabalı ters sarkaç ve top ve çubuk sistemi gibi laboratuvar cihazları eksik eyleyicili mekanik sistemlere birer örnek olarak kontrol yöntemlerinin test edilmesinde ve karşılaştırılmasında sıklıkla kullanılmaktadır [7,8].

Top ve çubuk sistemi doğrusal ve kararlı olmayan yapısıyla birçok klasik ve modern kontrol yönteminin test edilmesinde araştırmacılar tarafından tercih edilmektedir [9-10]. Ayrıca uçakların iniş sırasında yatay dengesinin sağlanması gibi gerçek kontrol problemleri de bu sistem sayesinde modellenebilmektedir [11]. Sistem açısı bir DC motor tarafından kontrol edilen bir çubuk ve bu çubuğun üzerinde kaymadan yuvarlanan bir toptan meydana gelmektedir. Dolayısıyla sistemde DC motorun çalışmasını sağlayan bir voltaj girişine karşılık çubuğun açısı ve topun konumunu ifade eden iki ayrı çıkış bulunmaktadır. Literatürde top ve çubuk sisteminin kontrolü için lineer kuadratik regülatör [12], PID [13], kayan kipli kontrol [14] ve geri adımlamalı kayan kipli kontrol [15] gibi birçok kontrol yaklaşımı önerilmiştir.

Geri adımlamalı kontrol yöntemi Lyapunov kararlılık teoremine dayanan bir kontrol yaklaşımıdır [16]. Burada sistemin kararlılık analizinde kullanılan Lyapunov fonksiyonu ve sistemi kontrol etmek için kullanılan kontrol kuralı özyinelemeli sistematik bir prosedür ile belirlenmektedir [17]. Kontrol kuralının belirlenmesi doğrudan Lyapunov teoremine dayandığı için sistemin kararlılığı da garanti altına alınmaktadır. Literatürde geri adımlamalı kontrol yönteminin insansız hava araçları [18], su altı araçları [19] ve robotik [20] gibi karmaş1k sistemlerin kontrolünde kullanıldığı görülmektedir. Ayrıca geri adımlamalı kontrol yöntemi belirsizliklere ve bozuculara karşı etkili kontrol kurallarının tasarlanmasında kayan kipli kontrol yöntemi ile birlikte de kullanılabilmektedir [21,22]

Uygun şartlar altında belirsizliklere ve bozuculara karşı etkili bir performans sağlayan bir yöntem olarak bilinen kayan kipli kontrol, değişken yapılı kontrol yönteminin özelleşmiş bir türüdür [23]. Geleneksel kayma kipli kontrol metodu erişme evresi ve kayma evresi olarak adlandırılan iki temel evreden oluşmaktadır. Erişim evresinde sistem durumları başlangıç konumlarından daha önceden tanımlanmış olan bir kayma yüzeyine doğru harekete zorlanmaktadır. Erişim evresi sistem durumlarının kayma yüzeyi üzerinde denge noktasına doğru hareket ettiği kayma evresi tarafından takip edilmektedir [24]. Sistem kayma yüzeyi üzerindeyken bozuculara karşı duyarsızdır [25].

Geleneksel kayan kipli kontrol yönteminin en önemli dezavantajlarından biri çatırdama problemi olarak adlandırılan kontrol sinyalindeki yüksek frekanslı salınım etkisidir [26]. Bir diğer dezavantaj ise bu yöntemin yüksek bağlaşık yapıları nedeniyle eksik eyleyicili mekanik sistemlere doğrudan uygulanamamasıdır. $\mathrm{Bu}$ sorunu aşmak için eksik eyleyicili mekanik sistemlerin kontrolünde ayrıklaştırılmış kayma kipli kontrol (AKKK) yöntemi kullanılabilmektedir [27]. AKKK yönteminin temel yaklaşımı sistemi iki alt sisteme ayırıp bunların her biri için farklı birer kayma yüzeyi tanımlamaktır. Dahası bu ayrıklaştırma yaklaşımı geri adımlamalı kayan kipli kontrol yöntemine de uygulanabilmektedir [28].

Bu çalışmada Ata ve Çoban [28] tarafından iki serbestlik derecesine sahip eksik eyleyicili mekanik sistemlerin kontrolü için önerilmiş olan ayrıklaştırılmış geri adımlamalı kayan kipli kontrol (AGKKK) yaklaşımı kullanılarak top ve çubuk sistemi için bir kontrolör tasarlanmıştır. Etkinlik ve performansının incelenebilmesi için yöntem 
benzetim çalışmalarıyla Lo ve Kuo [27] tarafından önerilen AKKK metodu ile karşılaştırılmış ve her iki yöntemin bozucu girişe karşı tepkisi incelenmiştir.

Çalışmanın 2. bölümünde benzetim çalışmalarında kullanılan top ve çubuk sisteminin matematiksel modeli tanıtılmış ardından ayrıklaştırılmış kayan kipli kontrol ve ayrıklaştırılmış geri adımlamalı kayan kipli kontrol yöntemleri ile kontrolör tasarım adımları ele alınmıştır. 3. bölümde benzetim çalışmaları ile elde edilen karşılaştırma bulgular sunulmuş, 4 . bölümde ise çalışmadan elde edilen sonuçlar ve gelecek çalışmalar için önerilere yer verilmiştir.

\section{MATERYAL VE METOT}

\subsection{Top ve Çubuk Sisteminin Matematiksel Modeli}

Top ve çubuk sistemi, bir top, topun üzerinde yer değiştirdiği bir çubuk ve bu çubuğu hareket ettiren bir DC motordan oluşan mekanik bir sistemdir. Top ve çubuk sisteminin parametrik gösterimi Şekil 1'de sunulmuştur. Çubuğun yatay düzlem ile yaptığı açı neticesinde top, çubuk ile temasını koruyarak yuvarlanmaktadır. Sistemin kontrol hedefi çubuğun açısı vasitasıyla topun konumunu denetlemektir. Dolayısıyla sistem biri çubuğun dairesel hareketi diğeri ise topun çubuk üzerindeki hareketi olmak üzere iki serbestlik derecesine sahiptir. Ancak bunlardan sadece çubuğun hareketi bir giriş sinyali ile denetlenebildiği için top ve çubuk sistemi eksik eyleyicili mekanik sistemlere bir örnek oluşturmaktadır.

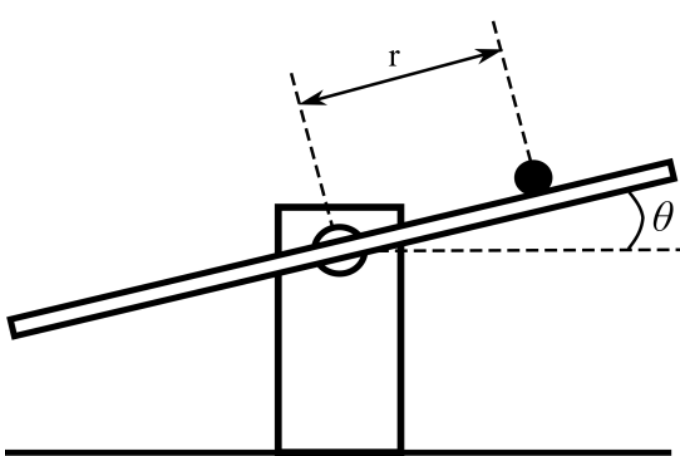

Şekil 1. Top ve çubuk sistemi
Çubuğun açısı $\theta$, topun konumu ise $r$ olarak ele alınırsa sistemin hareket denklemleri Eşitlik 1 ve 2'de verilen bağıntılar ile ifade edilebilir [29].

$\left(\frac{J_{C}}{R^{2}}+m\right) \ddot{r}+m g \sin (\theta)-m r \dot{\theta}^{2}=0$

$\left(m r^{2}+J_{C}+J_{T}\right) \ddot{\theta}+2 m r \dot{r} \dot{\theta}+m g r \cos (\theta)=\tau$

Burada $J_{C}$ çubuğun eylemsizlik momenti; $m, R$ ve $J_{T}$ sirasıyla topun kütlesi, çap1 ve eylemsizlik momenti; $g$ yer çekimi ivmesi ve $\tau$ ise çubuğa uygulanan kuvvet momentidir.

Top ve çubuk sistemine uygulanan kontrol sinyali $u$ ile çubuğa uygulanan kuvvet momenti $\tau$ arasında dönüşüm için Eşitlik 3'te verilen bağıntı kullanılabilir [29]:

$$
\tau=2 m r \dot{r} \dot{\theta}+m g r \cos (\theta)+\left(m r^{2}+J_{C}+J_{T}\right) u
$$

Sistemin durumlar1 $\left[\begin{array}{llll}\mathrm{x}_{1} & \mathrm{x}_{2} & \mathrm{x}_{3} & \mathrm{x}_{4}\end{array}\right]^{\mathrm{T}}=\left[\begin{array}{lll}\mathrm{r} & \dot{\mathrm{r}} \boldsymbol{\theta} & \dot{\theta}\end{array}\right]^{\mathrm{T}}$ olarak tanımlanırsa, Eşitlik 1 ve 2 'de verilen hareket denklemleri ile Essitlik 3'te verilen kontrol sinyali dönüşüm bağıntısı yardımıyla top ve çubuk sisteminin durum denklemleri Eşitlik 4'deki gibi tanımlanabilir [27,29]:

$$
\begin{aligned}
& \dot{x}_{1}(t)=x_{2}(t) \\
& \dot{x}_{2}(t)=\frac{M}{J_{T} / R^{2}+M}\left(x_{1}(t) x_{4}^{2}(t)-g \sin \left(x_{3}(t)\right)\right) \\
& \dot{x}_{3}(t)=x_{4}(t) \\
& \dot{x}_{4}(t)=u(t)+\epsilon(t)
\end{aligned}
$$

Burada $\epsilon(t)$ diş bozucuların ve belirsizliklerin toplamını ifade etmektedir ve $|\epsilon(t)| \leq \epsilon_{\max }$ ile sinırlı olduğu varsayılmaktadır.

\subsection{Ayrıklaştırılmış Kayan Kipli Kontrol}

Kayan kipli kontrol değişken yapılı kontrol yönteminin özel bir türüdür ve model belirsizlikleri ile dış bozuculara karşı gürbüz bir kontrol yöntemi olarak doğrusal olmayan sistemlerin kontrolünde sıklıkla kullanılmaktadır $[23,24]$. 
Klasik kayan kipli kontrol yönteminin temel iki evreden oluşmaktadır. Öncelikle kapalı çevrim sistemin tepkisine göre durum uzayında bir kayma yüzeyi tasarlanır. İlk evrede sonsuz anahtarlama frekansına sahip bir geri besleme sinyali vasıtasıyla sistem tepkisi bu yüzeye çekilir. İkinci evrede ise sistem tepkisi yüzey üzerinde tutularak denge noktasına doğru kaydırılır. Bu evreler erişme evresi ve kayma evresi olarak tanımlanmaktadır. İdeal kayan kipli kontrol yaklaşımında sistem, kayma yüzeyine ulaştıktan sonra bu yüzeyde kalır ve bozuculara karşı dirençli bir davranış ortaya koyar [23,24].

Klasik kayan kipli kontrol yöntemi kanonik formda ifade edilebilen sistemlerin kontrolü için uygundur ancak eksik eyleyicili sistemlere doğrudan uygulanamamaktadır. Top ve çubuk sistemi gibi 4. dereceden tek girişli eksik eyleyicili sistemler Eşitlik 5'teki gibi tanımlanabilir:

$$
\begin{aligned}
& \dot{x}_{1}(t)=x_{2}(t) \\
& \dot{x}_{2}(t)=\phi_{1}(x, t)+\gamma_{1}(x, t) u(t)+\epsilon_{1}(t) \\
& \dot{x}_{3}(t)=x_{4}(t) \\
& \dot{x}_{4}(t)=\phi_{2}(x, t)+\gamma_{2}(x, t) u(t)+\epsilon_{2}(t)
\end{aligned}
$$

Burada $\mathrm{x}=\left[\begin{array}{llll}\mathrm{x}_{1} & \mathrm{x}_{2} & \mathrm{x}_{3} & \mathrm{x}_{4}\end{array}\right]^{\mathrm{T}}$ durum vektörü; $\phi_{1}(\mathrm{x}, \mathrm{t})$, $\phi_{2}(\mathrm{x}, \mathrm{t}), \gamma_{1}(\mathrm{x}, \mathrm{t})$, ve $\gamma_{2}(x, t)$ doğrusal olmayan fonksiyonlar ve $\mathrm{u}(\mathrm{t})$ kontrol sinyalini ifade ederken $\epsilon_{1}(\mathrm{t})$ ve $\epsilon_{2}(\mathrm{t})$ belirsizlik ve bozucuların toplamını ifade etmektedir. Ayrica $\epsilon_{1}(t)$ ile $\epsilon_{2}(t)$ 'nin $\left|\epsilon_{1}(t)\right| \leq \epsilon_{1 \max }$ ve $\left|\epsilon_{2}(t)\right| \leq \epsilon_{2 \max }$ ile sinırlı olduğu ve $\epsilon_{1 \max }$ ve $\epsilon_{2 \max }$ 'in pozitif sabitler olduğu varsayılmaktadır.

Eşitlik 5'teki gibi eksik eyleyicili sistemlerin kayan kipli kontrolü için AKKK metodu kullanılabilir [27,30]. AKKK yönteminin temel yaklaşımı sistemi iki alt sisteme ayırıp bunların her biri için farklı birer kayma yüzeyi tanımlamaktır. Eşitlik 5'te verilen sistemin çıkışları $\mathrm{y}_{1}(\mathrm{t})=\mathrm{x}_{1}(\mathrm{t})$ ve $\mathrm{y}_{2}(\mathrm{t})=\mathrm{x}_{3}(\mathrm{t})$, beklenen ç1kışlar ise $\mathrm{y}_{\mathrm{d} 1}(\mathrm{t})$ ve $\mathrm{y}_{\mathrm{d} 2}(\mathrm{t})$ olarak tanımlandığında her iki çıkış için elde edilen izleme hataları ve kayma yüzeyleri Eşitlik 6-9' da verilmiştir.

$$
\begin{aligned}
& e_{1}(t)=y_{1}(t)-y_{d 1}(t) \\
& s_{1}(t)=k_{1} e_{1}(t)-\dot{e}_{1}(t) \\
& e_{2}(t)=y_{2}(t)-y_{d 2}(t) \\
& s_{2}(t)=k_{2} e_{2}(t)-\dot{e}_{2}(t)
\end{aligned}
$$

Burada $e_{1}(t)$ ve $e_{2}(t)$ izleme hatalari; $s_{1}(t)$ ve $s_{2}(t)$ kayma yüzeyleri; $\mathrm{k}_{1}$ ve $\mathrm{k}_{2}$ ise reel pozitif sabitlerdir.

Kayan kipli kontrol yönteminin temel amacı kayma yüzeyini sıfıra doğru yaklaştırmaktır. Bu nedenle kayma yüzeylerinden birinin değerini bir ara değişken yardımıyla diğer kayma yüzeyine aktararak her iki alt sistemi eş zamanlı olarak kontrol etmek mümkündür. Eşitlik 7'de verilen kayma yüzeyi $s_{1}(t)$ değerinden elde edilen transfer değişkeni $\mathrm{z}(\mathrm{t})$ ve bu değişken yardımıyla Eşitlik 9'da verilen kayma yüzeyi $s_{2}(t)$ 'nin ayrıklaştırılmış kayma yüzeyine dönüşüm bağıntıları sırasıyla Eşitlik 10 ve 11'de verilmiştir.

$z(t)=\operatorname{sat}\left(\frac{s_{1}(t)}{\Delta_{z}}\right) z_{u}, \quad 0<z_{u}<1$

$s_{2}(t)=k_{2}\left(e_{2}(t)-z(t)\right)+\dot{e}_{2}(t)$

Burada $z_{u}, z(t)$ 'nin üst limitini; $\Delta_{\mathrm{z}}$ ise sinır seviyesini ifade eden sabitlerdir. Satürasyon fonksiyonu sat( $\kappa)$ ise Eşitlik 12 ile tanımlanmıştır:

$\operatorname{sat}(\kappa)= \begin{cases}\operatorname{sign}(\kappa), & |\kappa| \geq 1 \\ \kappa, & |\kappa|<1\end{cases}$

Böylece $z(t)$ 'nin üst limiti $z_{-} u, s_{2}(t)$ 'nin sınırlı olmasını garanti altına alacak ve transfer değişkeni sayesinde $\mathrm{s}_{1}(\mathrm{t})$ 'nin sıfıra ulaşmasının ardından $\mathrm{s}_{2}(\mathrm{t})$ de sifira yakınsayacaktır.

Ayrıklaştırılmış kayan kipli kontrolör tasarımı için Lyapunov fonksiyonu ve bu fonksiyonun zaman bağlı türevi Eşitlik 13 ve 14'te verilmiştir. 


$$
V\left(s_{2}\right)=\frac{1}{2} s_{2}^{2}(t)
$$

$$
\begin{aligned}
\dot{V}\left(s_{2}\right) & =s_{2}(t) \dot{s}_{2}(t) \\
& =s_{2}(t)\left(k_{2}\left(\dot{e}_{2}(t)-\dot{z}(t)\right)+\ddot{e}_{2}(t)\right) \\
& =s_{2}(t)\left(k_{2}\left(\dot{e}_{2}(t)-\dot{z}(t)\right)\right) \\
& +s_{2}(t)\left(\ddot{y}_{2}(t)-\ddot{y}_{d 2}(t)\right) \\
& =s_{2}(t)\left(k_{2}\left(\dot{e}_{2}(t)-\dot{z}(t)\right)+\dot{x}_{4}-\ddot{y}_{d 2}(t)\right) \\
& =s_{2}(t)\left[k_{2}\left(\dot{e}_{2}(t)-\dot{z}(t)\right)\right. \\
& \left.+\phi_{2}(x)+\gamma_{2}(x) u_{S}(t)+\epsilon_{2}(t)-\ddot{y}_{d 2}(t)\right]
\end{aligned}
$$

Eşitlik 14'te verilen Lyapunov fonksiyonunun türevinin negatif tanımlı olabilmesi için gereken kontrol kuralı $\mathrm{u}_{\mathrm{S}}(\mathrm{t})$ Eşitlik 15'te verilmiştir.

$$
\begin{aligned}
u_{S}(t) & =\frac{1}{\gamma_{2}(x)}\left[-k_{2}\left(\dot{e}_{2}(t)-\dot{z}(t)\right)+\ddot{y}_{d 2}(t)\right] \\
& -\frac{1}{\gamma_{2}(x)}\left(\phi(x)+K \operatorname{sign}\left(s_{2}(t)\right)\right)
\end{aligned}
$$

Burada $K$ pozitif sabiti $K>\epsilon_{2 \max } \geq\left|\epsilon_{2}(t)\right|$ olmalıdır. Eşitlik 15'in Eşitlik 14'te yerine koyulmasıyla Eşitlik 16 elde edilir:

$$
\begin{aligned}
\dot{V}\left(s_{2}\right) & =s_{2}(t)\left(-K \operatorname{sign}\left(s_{2}(t)\right)+\epsilon_{2}(t)\right) \\
& =-K s_{2}(t) \operatorname{sign}\left(s_{2}(t)\right)+s_{2}(t) \epsilon_{2}(t) \\
& \leq-K\left|s_{2}(t)\right|+s_{2}(t) \epsilon_{2 \max } \\
& \leq-\left|s_{2}(t)\right|\left(K-\epsilon_{2 \max }\right) \\
& <0
\end{aligned}
$$

Lyapunov kararlılık teoremine göre $\dot{V}\left(s_{2}\right)$ negatif tanımlı olduğu için sistem kayma yüzeyine ulaşacak ve yüzey üzerinde kalacaktır. Böylece sistemin asimptotik kararlılığı garanti altına alınmıştır.

\subsection{Ayrıklaştırılmış Geri Adımlamalı Kayan Kipli Kontrol}

Geri adımlama yöntemi, kontrol kuralının belirlenmesinde yinelemeli Lyapunov kararlılık teoremini kullanan bir doğrusal olmayan kontrol metodudur [16]. Geri adımlamalı kontrol yöntemi, model belirsizliklerine ve diş bozuculara karşı etkili bir kontrol kuralının tasarlanmasında sıklıkla kayan kipli kontrol yöntemi ile birlikte kullanılmaktadır [31]. Ancak tıpkı kayan kipli kontrol yönteminde olduğu gibi geri adımlamalı kayan kipli kontrol yöntemi de kanonik formdaki sistemlere uygulanabilirken Eşitlik 5'te verilen eksik eyleyicili mekanik sistemlerin kontrolünde doğrudan kullanılamamaktadır. Bu nedenle eksik eyleyicili sistemlerin geri adımlamalı kayan kipli kontrolü için AGKKK yöntemi kullanılabilir [29].

Eşitlik 5'te verilen eksik eyleyicili sistemi için geri adımlamalı kayan kipli kontrol kuralı tasarımının ilk adımında Eşitlik 8'de verilen izleme hatası kullanılarak seçilen Lyapunov fonksiyonu ve bu fonksiyonun zamana bağlı türevi bağıntıları sırasıyla Eşitlik 17 ve 18'de verilmiştir.

$$
\begin{aligned}
V_{B 1}\left(e_{2}\right) & =\frac{1}{2} e_{2}^{2}(t) \\
\dot{V}_{B 1}\left(e_{2}\right) & =e_{2}(t) \dot{e}_{2}(t) \\
& =e_{2}(t)\left(x_{4}(t)-y_{d 2}(t)\right)
\end{aligned}
$$

Tasarımın bu aşamasında sistemin çıkışlarına karşılık gelen iki farklı kayma yüzeyi aşağıdaki gibi seçilebilir:

$s_{B 1}(t)=c_{1} e_{1}(t)+\dot{e}_{1}(t)$

$s_{B 2}(t)=c_{1} e_{2}(t)+\dot{e}_{2}(t)$

Burada $c_{1}$ ve $c_{2}$ pozitif sabitlerdir.

Sistem durumlarından $\mathrm{x}_{4}(\mathrm{t})$ sanal kontrol sinyali olarak seçilmiş ve Eşitlik 21 'de verilen bağıntı ile yeniden tanımlanmıştır.

$x_{4}(t)=s_{B 2}(t)-c_{2} e_{2}(t)+\dot{y}_{d 2}(t)$

Eşitlik 21'de verilen $x_{4}(t)$ 'nin Eşitlik 18' de yerine konulmasıyla Eşitlik 22 elde edilir:

$\dot{V}_{B 1}\left(e_{2}\right)=e_{2}(t) s_{B 2}(t)-c_{2} e_{2}^{2}(t)$ 
$\dot{\mathrm{V}}_{\mathrm{B} 1}\left(\mathrm{e}_{2}\right)=-\mathrm{c}_{2} \mathrm{e}_{2}^{2}(\mathrm{t}) \quad$ bağıntıs1 yalnızca $\quad \mathrm{s}_{\mathrm{B} 2}(\mathrm{t})=0$ olması durumunda negatif tanımlı olacaktır. $\mathrm{Bu}$ nedenle bir sonraki adımda kararlılığın garanti altına alınabilmesi için seçilen Lyapunov fonksiyonu Eşitlik 23'te verilmiştir.

$$
V_{B 2}\left(e_{2}\right)=V_{B 1}\left(e_{2}\right)+\frac{1}{2} s_{B 2}^{2}(t)
$$

Eşitlik 22'nin yardımıyla $V_{B 2}\left(e_{2}\right)$ nin zamana bağlı türevi Eşitlik 24'te elde edilmiştir.

$$
\begin{aligned}
\dot{V}_{B 2}\left(e_{2}\right) & =\dot{V}_{B 1}\left(e_{2}\right)+s_{B 2}(t) \dot{s}_{B 2}(t) \\
& =e_{2}(t) s_{B 2}(t)-c_{2} e_{2}^{2}(t) \\
& +s_{B 2}(t)\left(\phi_{2}(x)+\gamma_{2}(x) u_{B}(t)\right) \\
& +s_{B 2}(t)\left(\epsilon_{2}(t)+c_{2} \dot{e}_{2}(t)-\ddot{y}_{d 2}(t)\right)
\end{aligned}
$$

Burada $\mathrm{u}_{\mathrm{B}}(\mathrm{t})$ geri adımlamalı kayan kipli kontrol kuralıdır ve $\dot{\mathrm{V}}_{\mathrm{B} 2}\left(\mathrm{e}_{2}\right)$ 'nin negatif tanımlı olmasını sağlamak için tasarlanan kontrol kuralı Eşitlik 25' te verilmiştir.

$$
\begin{aligned}
u_{B}(t) & =\frac{1}{\gamma_{2}(x)}\left(-\phi_{2}(x)-e_{2}(t)-c_{2} \dot{e}_{2}(t)\right) \\
& +\frac{1}{\gamma_{2}(x)}\left(\ddot{y}_{d 2}(t)-c_{3} s_{B 2}(t)\right) \\
& -\frac{1}{\gamma_{2}(x)}\left(B \operatorname{sign}\left(s_{B 2}(t)\right)\right)
\end{aligned}
$$

Burada $c_{3}$ ve $B$ pozitif sabitlerdir. Ayrıca $B$ pozitif sabiti $B>\epsilon_{2 \max } \geq\left|\epsilon_{2}(\mathrm{t})\right|$ olacak şekilde seçilmelidir.

Eşitlik 25'in Eşitlik 24'te yerine koyulmasıyla Eşitlik 26 elde edilir:

$$
\begin{aligned}
\dot{V}_{B 2}\left(e_{2}\right) & =-c_{2} e_{2}^{2}(t)-c_{3} s_{B 2}^{2}(t) \\
& +\epsilon_{2}(t) s_{B 2}(t)-B\left|s_{B 2}(t)\right| \\
& \leq-c_{2} e_{2}^{2}(t)-c_{3} s_{D 2}{ }^{2}(t) \\
& -\left|s_{D 2}(t)\right|\left(B-\epsilon_{2 \max }\right) \\
& <0
\end{aligned}
$$

$B>\epsilon_{2 \max } \geq\left|\epsilon_{2}(t)\right| \quad$ koşulunun sağlanması durumunda $\dot{V}_{B 2}\left(e_{2}\right)$ negatif tanıml olacaktır. Böylece Lyapunov kararlılık teoremine göre sistemin asimptotik kararlılığı garanti altına alınmıştır.

Ayrıklaştırılmış bir kontrolör tasarlamak için Eşitlik 19'da verilen kayma yüzeyi $\mathrm{S}_{\mathrm{B} 1}(\mathrm{t})$ değerinden elde edilen transfer değişkeni $\mathrm{z}_{\mathrm{B}}(\mathrm{t})$ ve bu değişken yardımıyla tanımlanan ayrıklaştırılmış kayma yüzeyi bağıntıları sırasıyla Eşitlik 27 ve 28' de verilmiştir.

$$
\begin{aligned}
& z_{B}(t)=\operatorname{sat}\left(\frac{s_{B 1}(t)}{\Delta_{B z}}\right) z_{B u}, \quad 0<z_{B u}<1 \\
& s_{d}(t)=c_{2}\left(e_{2}(t)-z_{B}(t)\right)+\dot{e}_{2}(t)
\end{aligned}
$$

Burada $\mathrm{z}_{\mathrm{Bu}}, \mathrm{z}_{\mathrm{B}}(\mathrm{t})$ 'nin üst limitini; $\Delta_{\mathrm{Bz}}$ ise sınır seviyesini ifade eden sabitlerdir.

Sonuç olarak, Eşitlik 28'de verilen ayrıklaştırılmış kayma yüzeyinin Eşitlik 25'teki kayma yüzeyi $\mathrm{s}_{\mathrm{B} 2}(\mathrm{t})$ 'nin yerine koyulmasıyla elde edilen eksik eyleyicili sistemler için AGKKK kuralı Eşitlik 29'da verilmiştir.

$$
\begin{aligned}
u_{B}(t) & =\frac{1}{\gamma_{2}(x)}\left(-\phi_{2}(x)-e_{2}(t)-c_{2} \dot{e}_{2}(t)\right) \\
& +\frac{1}{\gamma_{2}(x)}\left(\ddot{y}_{d 2}(t)-c_{3} s_{d}(t)\right) \\
& -\frac{1}{\gamma_{2}(x)}\left(B \operatorname{sign}\left(s_{d}(t)\right)\right)
\end{aligned}
$$

\section{BENZETIM BULGULARI}

Eşitlik 15'te verilen AKKK ve Eşitlik 25'te verilen AGKKK yöntemlerini karş1laştırmak için benzetim testleri yapılmıştır. Eşitlik 4'te verilen top ve çubuk sisteminin kontrolü için her iki yöntem kullanılarak birer kontrolör tasarlanmış, çatırdama etkisini azaltmak için tasarım aşamasında sign( $\kappa)$ işaret fonksiyonu yerine sat( $($ ) fonksiyonu kullanılmıştır. Kontrol amacı topun konumu ve çubuğun açısını başlangıç 
durumlarından denge noktasına getirmek olarak belirlenmiştir. Tüm benzetim testlerinde başlangıç değerleri $\theta=60^{\circ}, \mathrm{r}=10 \mathrm{~cm}$ olarak seçilirken test süresi $20 \mathrm{~s}$, örnekleme aralığı $0,001 \mathrm{~s}$ olarak alınmıştır.

Benzetim testleri sırasında Eşitlik 4'te verilen top ve çubuk sistemi için sistem parametreleri $\mathrm{J}_{\mathrm{T}}=2 \times 10^{-6}, \mathrm{M}=0,05 \mathrm{~kg}, \mathrm{R}=0,01 \mathrm{~m}$ ve $\mathrm{g}=9,8 \mathrm{~m} / \mathrm{s}^{2}$ olarak seçilmiştir [27]. AKKK ve AGKKK yöntemleri için deneme ve yanılma yöntemiyle belirlenmiş olan parametreler ise Çizelge 1'de verilmiştir.

Çizelge 1. Kontrolör parametreleri

\begin{tabular}{|c|c|}
\hline Parametre & Değeri \\
\hline $\mathrm{k}_{1}, \mathrm{c}_{1}$ & 0,5 \\
\hline $\mathrm{k}_{2}, \mathrm{c}_{2}$ & 10 \\
\hline $\mathrm{c}_{3}$ & 20 \\
\hline $\mathrm{K}, \mathrm{B}$ & 10 \\
\hline $\mathrm{z}_{\mathrm{Su}}, \mathrm{z}_{\mathrm{Du}}$ & 0,94 \\
\hline$\Delta_{\mathrm{Sz}}, \Delta_{\mathrm{Bz}}$ & 5 \\
\hline
\end{tabular}

İlk benzetim testinde AKKK ve AGKKK yöntemleri Eşitlik 4'te verilen top ve çubuk sistemi üzerinde karşılaştırılmıștır. Bu teste sisteme etki eden bozucuların toplamı $\epsilon(t)=0$ olarak kabul edilmiş, dolayısıyla sisteme herhangi bir bozucu sinyal dahil edillmemiştir.

Başlangıç koşulları $\theta=60^{\circ}, \mathrm{r}=10 \mathrm{~cm}$ ve bozucuların toplamı $\epsilon(t)=0$ için topun konumu, çubuğun açısı ve kontrol sinyali sırasıyla Şekil 2-4'te verilmiştir.

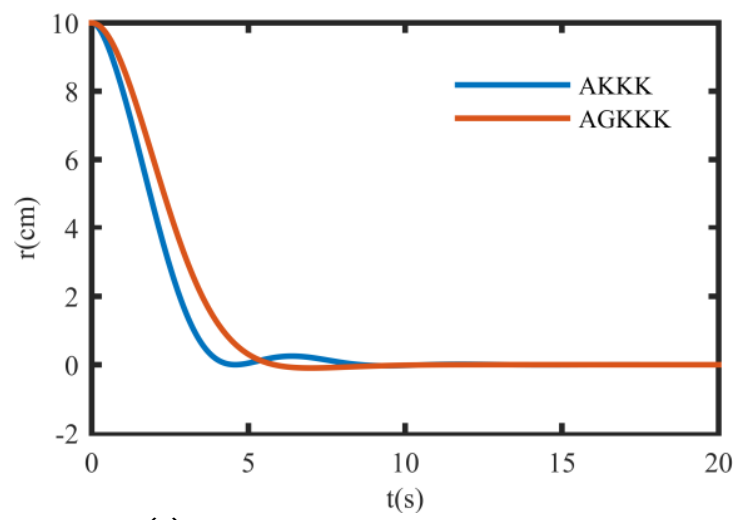

Şekil 2. $\epsilon(t)=0$ için topun konumu

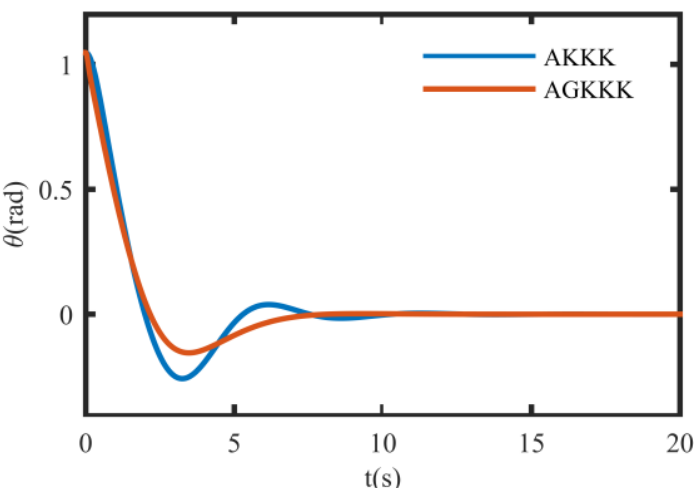

Şekil 3. $\epsilon(t)=0$ için çubuğun açısı

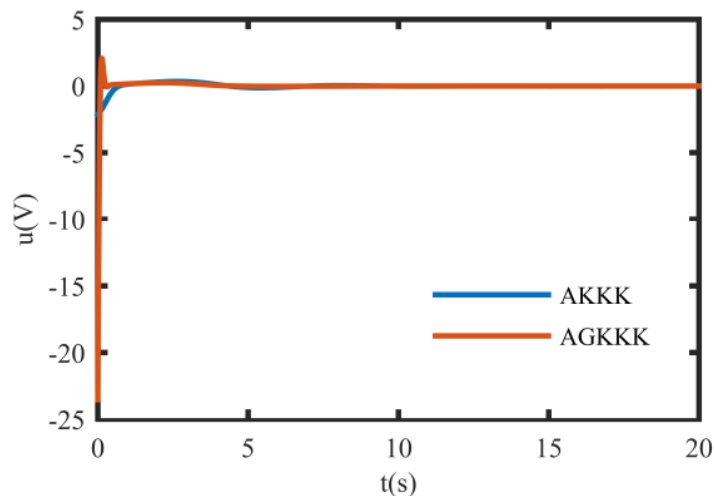

Şekil 4. $\epsilon(t)=0$ için kontrol sinyali

Şekil 2'de görüldüğü gibi her iki kontrolör de topun konumunu başarıyla kontrol etmektedir. Ancak AKKK için yerleşme zamanı 7,07 s iken AGKKK için 5,20 s olarak ölçülmüştür. $\mathrm{Bu}$ nedenle konum kontrolü için AGKKK'ün daha başarılı bir kontrol sinyali ürettiği görülmektedir.

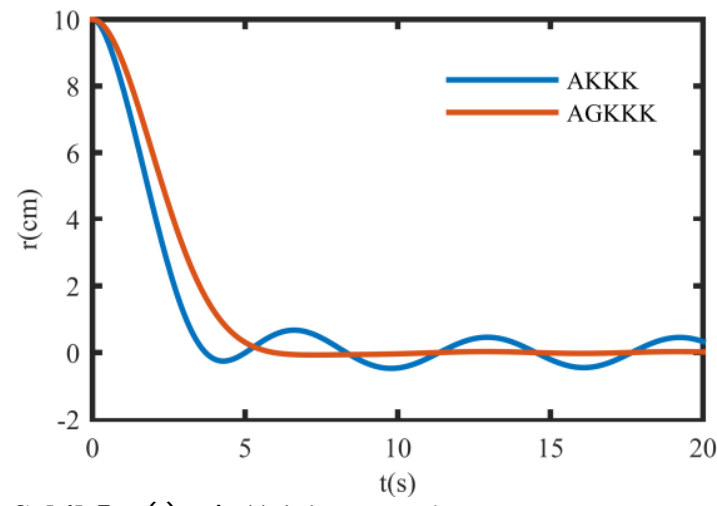

Şekil 5. $\epsilon(t)=\sin (t)$ için topun konumu 


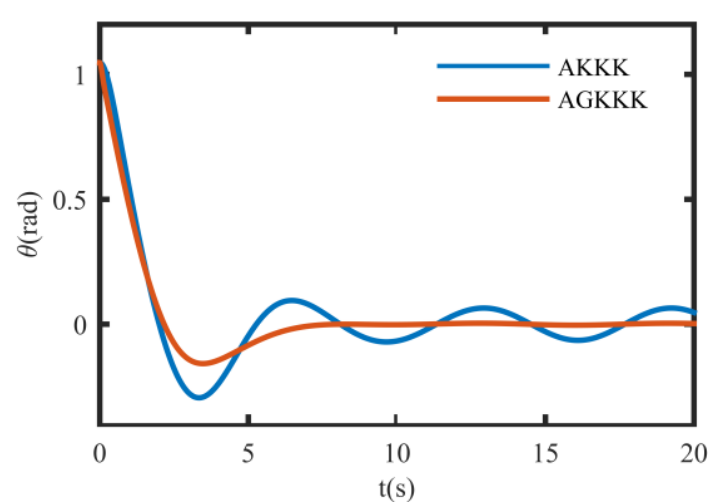

Şekil 6. $\epsilon(t)=\sin (t)$ için çubuğun açısı

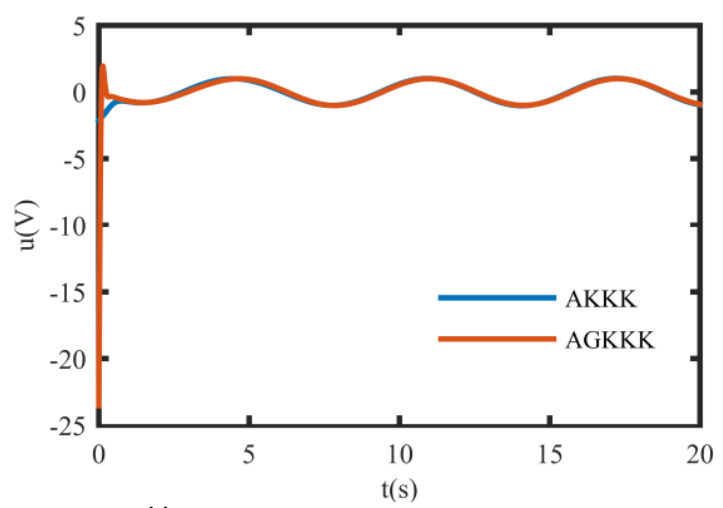

Şekil 7. $\epsilon(t)=\sin (t)$ için kontrol sinyali

Şekil 3 incelendiğinde her iki kontrolörün de yaklaşık olarak aynı zamanda çubuğun açısını kontrol etmeyi başardığg ancak AGKKK'nin daha düşük yüzde aşımı ile sistemi kontrol etmeyi başardığı görülmektedir.

İkinci benzetim testinde daha zorlayıı bir problem yaratmak için sisteme dış bozucu olarak $\epsilon(t)=\sin (t)$ büyüklüğünde bir sinyal dahil edilmiştir. Başlangıç koşulları $\theta=60^{\circ}, \mathrm{r}=10 \mathrm{~cm}$ ve bozucuların toplamı $\epsilon(t)=\sin (t)$ için topun konumu, çubuğun açısı ve kontrol sinyali sırasıyla Şekil 5-7'de verilmiştir.

Şekil 5 ve 6'da görüldüğü gibi artan diş bozucu etkisi ile birlikte AKKK hem topun pozisyonunu hem de çubuğun açısını kontrol etmekte başarısız olurken AGKKK dış bozuculara karşı etkili bir kontrol sinyali sunarak hem topun konumunu hem de çubuğun açısını stabilize etmeyi başarmıştır.

\section{SONUÇLAR}

$\mathrm{Bu}$ çalışmada eksik eyleyicili mekanik sistemlere örnek teşkil eden bir top ve çubuk sisteminin ayrıklaştırılmış geri adımlamalı kayan kipli kontrol (AGKKK) metodu ile bir denetleyici tasarlanmıştır. Tasarlanan denetleyicinin kararlılığın ve etkinliği benzetim çalışmaları ile incelenmiştir. Benzetim sonuçları dış bozucu etkisinin olmadiğı durumlarda AGKKK'nin geleneksel ayrıklaştırılmış kayan kipli kontrol (AKKK) yöntemine göre daha etkin bir kontrol sinyali oluşturduğunu ortaya koymuştur. Ayrica dış bozucu etkisi altında AKKK yöntemi sistemi kontrol etmede başarısız olurken AGKKK yöntemi hem topun pozisyonunu hem de çubuğun açısını başarıyla kontrol etmiş ve dış bozuculara karş1 etkili bir yöntem olduğunu göstermiştir.

Benzetim bulgularının tatmin edici sonuçlar ortaya koyması gelecek çalışmalarda bu yöntemin fiziksel bir top ve çubuk sisteminin kontrolünde kullanılabileceğini göstermiştir. Ayrıca bu yöntemin farklı eksik eyleyicili mekanik sistemler üzerinde kullanılması mümkündür. Bu çalışmada kontrolör parametreleri deneme yanılma metodu ile belirlenmiştir. AGKKK parametrelerinin belirlenmesinde çeşitli optimizasyon tekniklerinin kullanılması da gelecek çalışmalar için ilgi çekici bir başlık olacaktır.

\section{KAYNAKLAR}

1. Oryschuk, P., Salerno, A., Al-Husseini, A.M., Angeles, J., 2009. Experimental Validation of an Underactuated Two-wheeled Mobile Robot. IEEE/ASME Transactions on Mechatronics, 14(2), 252-257.

2. Woods, S.A., Bauer, R.J., Seto, M.L., 2012. Automated Ballast Tank Control System for Autonomous Underwater Vehicles. IEEE Journal of Oceanic Engineering, 37(4), 727-739.

3. Olfati-Saber, R., 2001. Nonlinear Control of Underactuated Mechanical Systems with Application to Robotics and Aerospace Vehicles. Doktora Tezi, Massachusetts Institute 
of Technology. Dept. of Electrical Engineering and Computer Science, 316.

4. Choukchou-Braham, A., Cherki, B., Djemaï, M., Busawon, K., 2013. Analysis and Control of Underactuated Mechanical Systems. Springer-Verlag, New York.

5. Liu, Y., Yu, H., 2013. A Survey of Underactuated Mechanical Systems. IET Control Theory and Applications, 7(7), 921-935.

6. Huang, X., Ralescu, A. L., Gao, H., Huang, H., 2019. A Survey on the Application of Fuzzy Systems for Underactuated Systems. Journal of Systems and Control Engineering, 233(3), 217-244.

7. She, J., Zhang, A., Lai, X., Wu, M., 2012. Global Stabilization of 2-DOF Underactuated Mechanical Systems-an Equivalent-inputdisturbance Approach. Nonlinear Dynamics, 69(1-2), 495-509.

8. Shah, I., Rehman, F.U., 2018. Smooth Second Order Sliding Mode Control of a Class of Underactuated Mechanical Systems. IEEE Access, 6(c), 7759-7771.

9. Mehedi, I.M., Al-Saggaf, U.M., Mansouri, R., Bettayeb, M., 2019. Two Degrees of Freedom Fractional Controller Design: Application to the Ball and Beam System. Measurement: Journal of the International Measurement Confederation, 135, 13-22.

10. Yang, J., Wang, Z.H., 2010. Adaptive Robust Dissipative Design on Stability Control for Ball and Beam System. International Conference on Computer and Automation Engineering, ICCAE 2010, 4, 68-71.

11. Guinaldo, M., Vargas, H., Sánchez, J., Sanz, E., Dormido, S., 2009. Web-based Control Laboratory: The Ball and Beam System. IFAC Proceedings Volumes 8, 174-179.

12. Choudhary, M.K., Naresh Kumar, G., 2016. ESO Based LQR Controller for Ball and Beam System. IFAC-PapersOnLine, 49(1), 607-610.

13. Hirschorn, R.M., 2002. Incremental Sliding Mode Control of the Ball and Beam. IEEE Transactions on Automatic Control, 47(10), 1696-1700.

14. Meenakshipriya, B., Kalpana, K., 2014. Modelling and Control of Ball and Beam System Using Coefficient Diagram Method
(CDM) Based PID Controller. IFAC Proceedings Volumes, 3, 620-626.

15. Moezi, S. A., Zakeri, E., Eghtesad, M., 2019. Optimal Adaptive Interval Type-2 Fuzzy Fractional-order Backstepping Sliding Mode Control Method for Some Classes of Nonlinear Systems. ISA Transactions, 93, 23-39.

16. Freeman, R.A., Kokotović, P., 1996. Robust Nonlinear Control Design. Birkhäuser, Boston.

17. Krstic, M., Kokotovic, P.V., Kanellakopoulos, I., 1995. Nonlinear and Adaptive Control Design. Wiley, New York.

18. Madani, T., Benallegue, A., 2006. Backstepping Control for a Quadrotor Helicopter. IEEE International Conference on Intelligent Robots and Systems, 3255-3260.

19. Liu, S., Liu, Y., Wang, N., Liu, S., Liu, Y., Wang, N., Wang, N., 2017. Nonlinear Disturbance Observer-based Backstepping Finite-time Sliding Mode Tracking Control of Underwater Vehicles with System Uncertainties and External Disturbances. Nonlinear Dynamics, 88, 465-476.

20. Pan, Y., Wang, H., Li, X., Yu, H., 2018. Adaptive Command-Filtered Backstepping Control of Robot Arms with Compliant Actuators. IEEE Transactions on Control Systems Technology, 26(3), 1149-1156.

21. Bouabdallah, S., Siegwart, R., 2005. Backstepping and Sliding-mode Techniques Applied to an Indoor Micro Quadrotor. IEEE International Conference on Robotics and Automation, 2247-2252.

22. Coban, R., 2017. Backstepping Sliding Mode Tracking Controller Design and Experimental Application to an Electromechanical System. Control Engineering and Applied Informatics, 19(3), 88-96.

23. Utkin, V., 1977. Variable Structure Systems with Sliding Modes. IEEE Transactions on Automatic Control, 22(2), 212-222.

24. Utkin, V., 1992. Sliding Modes in Control and Optimization. Springer, Berlin.

25. Edwards, C., Spurgeon, S., 1998. Sliding Mode Control. CRC Press, London.

26. Lee, H., Utkin, V.I., 2007. Chattering Suppression Methods in Sliding Mode Control Systems. Annual Reviews in Control, 31(2), 179-188. 
27. Lo, J. C., Kuo, Y. H., 1998. Decoupled Fuzzy Sliding-mode Control. IEEE Transactions on Fuzzy Systems, 6(3), 426-435.

28. Ata, B., Coban, R., 2019. Decoupled Backstepping Sliding Mode Control of Underactuated Systems with Uncertainty: Experimental Results. Arabian Journal for Science and Engineering, 44(8), 7013-7021.

29. Hauser, J., Sastry, S., Kokotovic, P., 1992. Nonlinear Control Via Approximate Inputoutput Linearization: The Ball and Beam Example. IEEE Transactions on Automatic Control, 37(3), 392-398.

30. Coban, R., Ata, B., 2017. Decoupled Sliding Mode Control of an Inverted Pendulum on a Cart: An Experimental Study. 2017 IEEE International Conference on Advanced Intelligent Mechatronics, 993-997.

31. Lu, C.H., Hwang, Y.R., Shen, Y.T., 2011. Backstepping Sliding Mode Tracking Control of a Vane-type Air Motor X-Y Table Motion System. ISA Transactions, 50(2), 278-286. 